\title{
Herramientas para la mejora de la práctica docente de la Informática. Experiencia piloto en el Grado en Gestión y Administración Pública.
}

\section{Francisco Mata ${ }^{a}$, Luis Gonzaga Pérez ${ }^{b}$ y Carlos Porcel ${ }^{c}$}

a Departamento de Informática, Universidad de Jaén, email: fmata@ujaen.es, bepartamento de Informática, Universidad de Jaén, email: lgonzaga@ujaen.es 'Departamento de Informática, Universidad de Jaén, email: cporcel@ujaen.es.

\begin{abstract}
In this paper we introduce the teaching innovation initiative based on the use of concept maps and cooperative learning for teaching computer science in non-technical degrees, in particular in Management and Public Administration Degree. The combination of both methodologies gather peculiarities we believe useful for students of this degree or similar degrees, improving skills (teamwork, responsibility and leadership) and academic results. It is a pilot experience that is under development currently. According first results, we believe it will be a successful case. Although we have focused on a specific discipline and degree, this experience may be extended or adapted to other disciplines with complex contents or unsatisfactory academic results.
\end{abstract}

Keywords: concept maps, cooperative learning, computer science.

\begin{abstract}
Resumen
En este trabajo presentamos la iniciativa de innovación docente basada en el uso de mapas conceptuales y aprendizaje cooperativo para la enseñanza de la informática en titulaciones no técnicas, en particular en el Grado en Gestión y Administración Pública. Ambas metodologías reúnen peculiaridades que creemos que pueden se útiles para los estudiantes de esta titulación y titulaciones similares, mejorando sus competencias (trabajo en equipo, responsabilidad y liderazgo) y resultados académicos. Se trata de una experiencia piloto que actualmente está en fase de desarrollo y que a tenor de los primeros resultados creemos que va a ser un éxito. Si bien nos hemos centrado en una disciplina y titulación en concreto, consideramos que la experiencia puede ser exportada y/o adaptada a otras disciplinas con un contenido complejo o con resultados académicos poco satisfactorios.
\end{abstract}

Palabras clave: mapas conceptuales, aprendizaje cooperativo, informática. 


\section{Introducción}

En la literatura podemos encontrar numerosas definiciones sobre el concepto de Innovación Docente, siendo todas ellas coincidentes básicamente en la idea de aplicar nuevas herramientas y metodologías docentes con el propósito de mejorar la calidad de la docencia impartida.

En línea con las ideas recogidas en el preámbulo y objetivos del Plan de Innovación Docente de la Universidad de Jaén (I2D-UJA, 2016) en lo que concierne a "una mejora de la calidad docente, la adquisición de competencias y los resultados académicos del alumnado de la Universidad de Jaén", en este trabajo presentamos dos iniciativas de innovación docente, mapas conceptuales y el aprendizaje cooperativo, que proponemos incorporar a nuestra práctica docente de la informática en el grado en Gestión y Administración Pública con el propósito de mejorar las competencias de nuestros estudiantes y en consecuencia sus resultados académicos.

Enseñar informática en titulaciones no técnicas requiere adaptar los contenidos y las estrategias de aprendizaje al perfil de los alumnos que en muchos casos ven la informática con recelo al considerarla fuera de su ámbito de estudio actual e incluso futuro. Si embargo, creo que estaremos todos de acuerdo, la informática, no desde el punto de vista del desarrollo de aplicaciones sino desde el punto de vista del uso de un ordenador de forma eficiente, es una destreza que ningún alumno, independientemente de titulación debería descuidar. En titulaciones técnicas esta disciplina se ha implantado sin demasiados problemas y ha sido rápidamente asumida como parte esencial de su curriculum por el alumnado. Sin embargo, en otras titulaciones, como es el caso de que aquí tratamos, su impartición resulta compleja y de ahí que los resultados académicos no sean los esperados. En nuestro caso concreto venimos impartiendo la docencia utilizando una metodología de enseñanza clásica basada en clases magistrales en gran grupo y clases prácticas en aulas de informática. Los alumnos ponen en práctica una estrategia clásica de aprendizaje memorístico que no obtiene los resultados esperados porque son incapaces de establecer relaciones entre los conceptos si previamente no han sido aprendidos, es decir, aprenden de memoria conceptos y definiciones pero sin tener claro su significado ni relaciones entre los mismos. Este tipo de aprendizaje que, para materias como legislación o similares puede ser válido, en nuestro caso consideramos que no lo es.

Partiendo de esta reflexión y haciendo nuestra la frase del creador del proyecto de Wikipedia Jimmy Wales “aprender a aprender" (Wales, 2018), nos hemos planteado cambiar el modelo de aprendizaje actual hacia un modelo en el que los alumnos, partiendo de unos conocimientos básicos, sean capaces de generar nuevo conocimiento, tanto de forma individualizada como trabajando en grupo. Para conseguirlo estamos desarrollando una experiencia piloto basada en el uso de mapas conceptuales y aprendizaje cooperativo. Ambos conceptos se introducen en la siguiente sección, dedicando el resto del trabajo a la presentación de los objetivos del proyecto, la metodología para ponerlo en marcha y los resultados esperados.

(cc) BY-NC-ND 2018, Universitat Politécnica de Valencia

Congreso IN-RED (2018) 


\subsection{Mapas conceptuales}

Los mapas conceptuales fueron propuestos por Novak (Novak, 1990) a raíz de un proyecto de investigación sobre el aprendizaje de la ciencias en niños. Tomando como referencia las propuestas del psicólogo estadounidense David Ausubel (Ausubel, 1968), Novak planteó representar el conocimiento de los niños de una forma gráfica, dando lugar a lo que hoy en día se conoce por mapas conceptuales (Novak \& Musonda, 1991).

Los mapas conceptuales son una herramienta de aprendizaje basada en la representación gráfica de conceptos y relaciones entre ellos, que apoyándose en una estructura jerárquica o de tipo grafo, permite una estructuración y organización del conocimiento. Partiendo de conceptos generales en los niveles superiores de la estructura, se puede ir descendiendo en la búsqueda de conceptos más específicos los cuales están enlazados por palabras o verbos significativos que representan relaciones entre los elementos enlazados. Se sustentan en dos elementos principales, i) conceptos, representados normalmente por cajas, y ii) relaciones, representadas por líneas que unen conceptos y están etiquetadas con proposiciones que describen el tipo de relación entre conceptos. En la Figura 1 se muestra un ejemplo de mapa conceptual sobre las estaciones del año y su relaciones con conceptos como la cantidad de luz solar y la altura del sol.

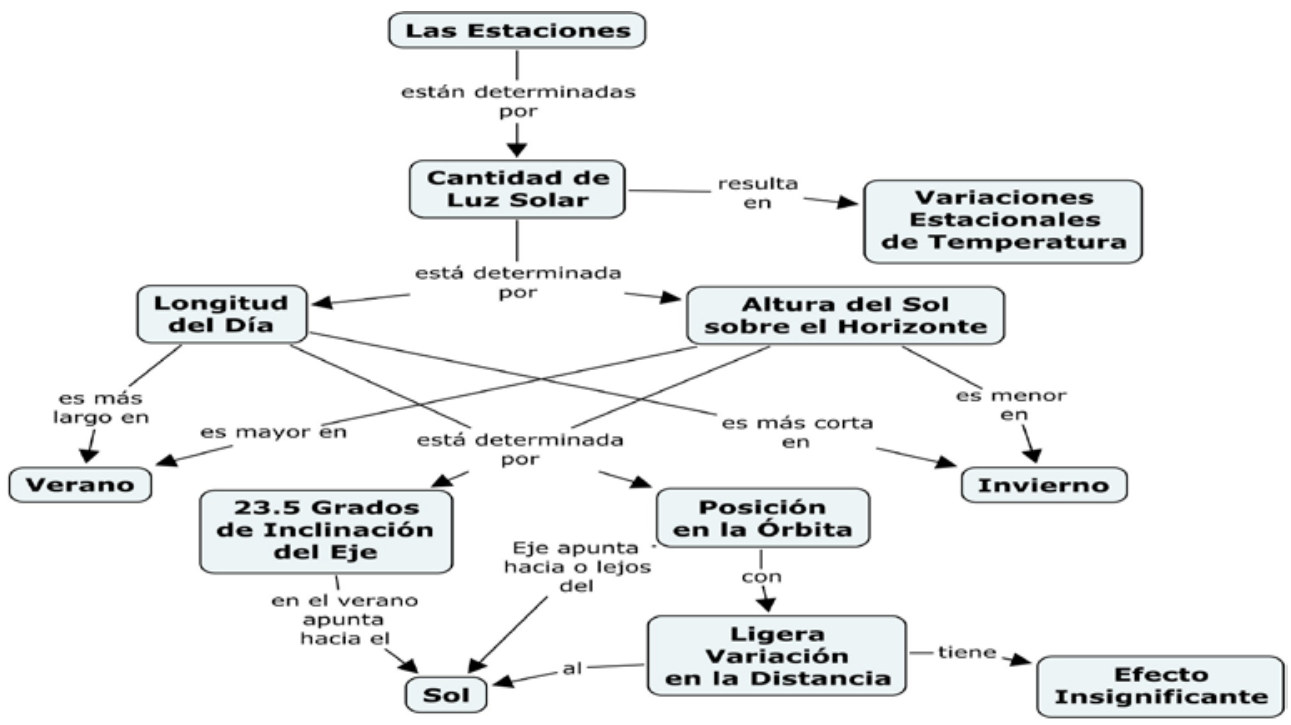

Fig. 1 Ejemplo de mapa conceptual ${ }^{1}$

\footnotetext{
${ }^{1}$ Fuente, https://cmap.ihmc.us/docs/images/Theory/spanish/Fig5CmapEstaciones-gr.jpg
} 
De forma general, el recorrido de un mapa conceptual comienza por el nodo superior de la jerarquía, descendiendo a continuación por cada una de las ramas. Cada rama representa una especificación del nodo superior, existiendo la posibilidad de establecer relaciones entre conceptos de ramas diferentes (enlaces cruzados), favoreciendo de esta forma la creatividad y la creación de nuevo conocimiento. Dependiendo del tema, los últimos nodos de la estructura jerárquica pueden ser en algunos casos ejemplos concretos sobre el concepto predecesor que ayudan a aclararlo.

Los mapas conceptuales son considerados como un ejemplo de aprendizaje significativo (Ausubel, 1963) basado en la idea del aprendizaje por descubriendo frente al aprendizaje por memorización (Tamayo, 2006). El aprendizaje significativo, a diferencia del memorístico basado en la idea de memorizar datos y conceptos, se basa en la idea de descubrir nuevos conceptos y relaciones, i.e. inferir nuevo conocimiento, a partir de la organización y estructuración del conocimiento previo y la incorporación de nuevos conceptos. Se puede decir que es la búsqueda de nuevos contenidos a partir del establecimiento de relaciones con el conocimiento del que ya se dispone.

Una de las ventajas principales del aprendizaje significativo y su implementación con mapas conceptuales es la representación estructurada de conocimiento de un individuo que, debidamente motivado, es capaz de construir nuevo conocimiento. Por lo tanto, la combinación de ambas estrategias pueden incrementar significativamente la creatividad de los individuos, en nuestro caso de los estudiantes.

Groso modo, el aprendizaje a través de mapas conceptuales se puede enfocar al menos desde dos puntos de vista.

a) Aprendizaje que se consigue cuando es el alumno el encargado de crear el mapa conceptual. Los expertos coinciden en que posiblemente esta es la mayor ventaja de los mapas conceptuales, pues obliga a que el sujeto tome una actitud activa en la búsqueda información, identificando nuevos conceptos y relaciones, y genere el mapa conceptual. Esto implica que se ejercite la parte creativa del estudiante y que un mismo problema sea enfocado de forma diferente por cada individuo. Pero por el contrario, requiere que el alumno tengan unos conocimientos mínimos que les permitan generar un estructura básica de conocimiento y a partir de esta, inferir el nuevo conocimiento.

b) Aprendizaje a partir de "mapas esqueleto" construidos por expertos (O'Donnell, et al., 2002). Este tipo de enfoque está pensado para aquellos casos en los que se aborden temas nuevos y/o complejos y alumno carezca de conocimientos y experiencia suficiente. La idea es partir de un mapa conceptual básico desarrollado por un experto y a partir de este, el estudiante ha de seguir construyéndolo incorporando nuevos conceptos y relaciones.

En la experiencia piloto que aquí estamos presentado estamos aplicando este segundo enfoque debido a que nuestros alumnos no tienen conocimientos ni experiencia para generar un mapa conceptual desde cero.

(cc) EY-NC-ND 2018, Universitat Politécnica de Valencia

Congreso IN-RED (2018) 


\subsection{Aprendizaje cooperativo}

$\mathrm{Al}$ igual que ocurre con el aprendizaje significativo, el aprendizaje cooperativo tiene su origen en la teoría constructivista propuesta por Piaget en la que el alumno juega un papel principal y activo en el proceso de aprendizaje (Piaget, 1973). El aprendizaje cooperativo, también conocido como colaborativo, se puede definir como una técnica de aprendizaje en la que los alumnos trabajan por grupos para alcanzar unos objetivos comunes, siendo responsables de su consecución todos los miembros del grupo (Servicio de Innovación Educativa, 2008). Johnson lo define cooperativo como aquel aprendizaje que persigue que los alumnos trabajen juntos para lograr objetivos que sean beneficiosos para todos los miembros del grupo (Johnson \& Johnson, 1991).

En la docencia en general y en la universitaria en particular es frecuente poner en práctica lo que los expertos denominan aprendizaje individualista, en el que el alumno se centra únicamente en la realización de su tarea y en conseguir, a nivel individual, sus objetivos previstos. Por tanto, el hecho de que un estudiante consiga de forma individual sus objetivos no influye en que sus compañeros también los alcancen. El aprendizaje individualista puede fácilmente derivar hacia otro tipo de aprendizaje denominado competitivo donde, además de trabajar de forma individualizada, se introduce el concepto de competitividad. Este concepto se traduce en el hecho de que alcanzar unos objetivos, en muchas ocasiones, implica la no consecución de esos objetivos por el resto de estudiantes (Garcia, et al., 2001). Según Prieto (Prieto, 2007), cada uno de los tipos de aprendizaje tiene sus pros y sus contras. Lo ideal sería aplicar un tipo de aprendizaje u otro en función del objetivo que se persiga y del contexto en el que se produzca el aprendizaje. Así, hay situaciones en las que se requiere que el estudiante trabaje de forma individual y otras en las que se requiere trabajar en grupo.

Centrándonos en el aprendizaje cooperativo, Johnson señala cinco características principales (Johnson, et al., 1999):

a) Interdependencia positiva. El éxito del grupo depende del éxito de cada uno de los miembros del grupo. Esta interdependencia implica la necesidad de apoyo entre todos los miembros del grupo.

b) Interacción "bis a bis”. Implica que los alumnos deben compartir conocimientos, recursos e interactuar entre sí para alcanzar el objetivo común.

c) Responsabilidad individual. Cada alumno debe ser responsable de llevar a cabo correctamente su tarea asignada y asumir tanto el éxito como el fracaso en el objetivo común del grupo.

d) Habilidades sociales. Permite poner en práctica las habilidades sociales de cada uno de los miembros del grupo desde el punto de vista de liderazgo y resolución de conflictos.

e) Autoevaluación. Es importante que el grupo realice una fase de autocrítica donde entre a valorar el funcionamiento del grupo y el grado de consecución de sus objetivos tanto a nivel individual como colectivo. Esta es la forma de aprender de los errores y plantearse nuevas estrategias para trabajos futuros.

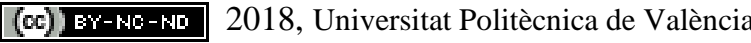

Congreso In-Red (2018) 
Herramientas para la mejora de la práctica docente de la Informática. Experiencia piloto en el Grado en Gestión y Administración Pública

\section{Contexto de la propuesta de innovación.}

Antes de enumerar los objetivos principales de esta experiencia piloto es necesario definir el contexto académico en el que se está llevando a cabo la misma. Este trabajo describe las líneas de actuación del proyecto de innovación docente titulado "Herramientas para la mejora de la práctica docente en la asignatura Introducción a la Informática de Gestión, grado en Gestión y Administración Pública”, encuadrado dentro del Plan de Innovación Docente de la Universidad de Jaén.

Hemos de tener en cuenta que transmitir conocimientos y competencias de la informática para alumnos cuyo perfil e interés en esta materia es escaso resulta muchas veces complicado a la par que desalentador tanto para el profesorado como para el propio estudiante. Aunque estamos notando cambios en los últimos años, todavía para muchos de los alumnos de titulaciones no técnicas, la informática les genera cierta desazón e incluso rechazo.

La experiencia piloto se está llevando a cabo sobre la asignatura Informática Aplicada a la Gestión, del Grado en Gestión y Administración Pública. Esta asignatura se imparte en el primer curso, primer cuatrimestre, con una carga teórica de 3 créditos y una carga práctica de otros 3. El número de alumnos por curso se mueve entre 20 y 50 alumnos lo que, según nuestra opinión, permite poner en práctica esta prueba piloto. El contenido está planteado como una primera toma de contacto con la disciplina de la informática desde el punto de vista de usuario de ordenadores y aplicaciones informáticas. Contempla conceptos generales de la Informática (software, hardware, Internet, Software Libre,...), destacando su parte práctica donde el alumno debe manejar de forma correcta y eficiente un equipo informático y un conjunto de aplicaciones (paquete de ofimática Open Office, navegadores web, etc). Estos contenidos se amplían en una segunda asignatura, Informática aplicada a la Administración Pública, que se imparte en el segundo cuatrimestre del mismo curso.

Los alumnos disponen de apuntes elaborados por el profesorado en formato libro. Desde el punto de vista metodológico, seguimos una metodología tradicional basada en clases magistrales en gran grupo apoyadas en recursos multimedia (presentaciones Power Point, videos,...) y evaluación con examen tanto teórico como práctico. Desde el punto de vista de los resultados medidos en número de aprobados y suspensos, aproximadamente el $30 \%$ no supera la asignatura, teniendo unos resultado peores que el resto de asignaturas del mismo curso, circunstancia que nos preocupa y que ha llevado entre otras cosas a poner en marcha esta iniciativa de innovación docente.

\section{Objetivos}

Una vez introducidos los aspectos más relevantes de las herramientas de innovación que vamos a poner en práctica y el contexto donde se está llevando a cabo la experiencia piloto, describiremos los problemas que hemos observado en la impartición de esta materia y plantearemos los objetivos a cubrir en el proyecto de innovación docente.

Problemas:

(cc) EY-NC-ND 2018, Universitat Politécnica de Valencia

Congreso IN-RED (2018) 
a) Aunque existe un material en formato libro elaborado por el profesorado y en continua actualización, el contenido teórico es exigente y requiere un trabajo diario del alumnado que hemos comprobado que no se realiza. Este material se elaboró para evitar que el alumno tuviese que tomar apuntes de unos contenidos completamente desconocidos y que en muchas ocasiones implicaba exámenes pobres y con muchas inexactitudes. Este material, que en principio puede verse como algo positivo puesto que recoge los contenidos teóricos de forma precisa, produce que un número importante de alumnos dejen de asistir a clase y confíen el aprobado al estudio memorístico del material suministrado.

b) Por diferentes motivos (selectividad en septiembre, cambios de universidad, etc), parte del alumnado se incorpora tarde a clase, cuando varios de los temas teóricos y prácticas ya se hayan impartido; consecuencia, les resulta complicado reengancharse a la asignatura y seguir el ritmo de las clases prácticas.

c) A esta titulación acceden alumnos con perfiles muy diferentes y con interés muy diferenciados. Alumnos que tienen más o menos claro el perfil de la titulación, alumnos de cierta edad que provienen de escalas básicas de la administración y que buscan con esta titulación una posible promoción interna y alumnos que no han sido admitidos en otras titulaciones y que finalmente se decantan por esta. Esta amalgama de estudiantes implica desequilibrios desde el punto de vista de la motivación y el interés en la materia así como en el dominio y destrezas en el uso de equipos informáticos.

Teniendo presente estos problemas, nuestra propuesta de mejora de la enseñanza de la informática en esta titulación persigue dos objetivos principales:

a) Para facilitar la asimilación de contenidos, la motivación y la creatividad de nuestros estudiantes, proponemos usar como herramienta de aprendizaje los mapas conceptuales. En concreto, el profesorado facilitará al alumnado un conjunto de mapas conceptuales esqueleto o básicos que los estudiantes deben usar como punto de partida para ampliar y mejorar su contenido. De esta forma el estudiante se convertirá en un agente activo en el proceso de aprendizaje y asimilará los conceptos a partir de la generación de nuevos contenidos y conocimiento.

b) Para evitar que, tanto el alumnado que se incorpora tarde o como el que tiene problemas en el uso de ordenadores y/o aplicaciones informáticas se pueda quedar descolgado en la parte práctica de la asignatura, pondremos en marcha un modelo de aprendizaje cooperativo conforme al paradigma introducido anteriormente. Tal y como se indica en el siguiente apartado, se han creado pequeños grupos de estudiantes con el objetivo común de colaborar y apoyarse entre sí en las clases prácticas con ordenadores.

Con la puesta en práctica de ambas estrategias de aprendizaje esperamos potenciar competencias tan importantes como el trabajo en equipo, creatividad y liderazgo. Como consecuencia, esperamos mejorar los resultados académicos de nuestros estudiantes.

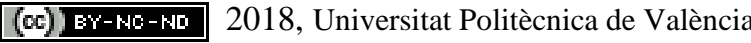


Herramientas para la mejora de la práctica docente de la Informática. Experiencia piloto en el Grado en Gestión y Administración Pública

\section{Desarrollo}

Fijados los objetivos, en este apartado comentaremos las estrategias y tareas que vamos a utilizar para alcanzar los objetivos propuestos.

\subsection{Mapas conceptuales.}

Nuestro propósito es realizar mapas conceptuales del temario teórico de la asignatura. Antes de incorporar el ordenador como una herramienta de trabajo, los mapas se solían dibujar a mano alzada o bien se utilizaban otro tipo de recursos como post-its, que se desplegaban sobre algún tipo de soporte que permitiese su desplazamiento, por ejemplo una pizarra. Actualmente existen herramientas informáticas, más o menos especializadas, para crear mapas conceptuales que se almacenan en formatos digitales que permiten su reproducción en equipos informáticos (ordenadores, tablets, smartphones,...) y su distribución a través de la red. Tras una revisión de varias aplicaciones software (Xmind, Inspiration, SmartDraw), nos hemos decantado por usar la aplicación CmapTools desarrollada por Florida Institute for Human \& Machine Cognition, IHMC (Cañas, et al., 2004). Esta herramienta puede crear mapas conceptuales tanto en modo local como en la nube, permitiendo la interacción entre individuos en diferentes ubicaciones físicas. Además permite, no solo representar conceptos y relaciones, sino incorporar otros elementos multimedia (imagen, sonido, video) y distribuirlos y compartillos por Internet. Otra de las ventajas de esta herramienta es la posibilidad de buscar información o recursos en internet relacionados y filtrados por los conceptos recogidos en mapa conceptual. Esto permite encontrar nueva información que se puede ir incorporando al mapa conceptual de forma relativamente sencilla. Por último añadir que este software se distribuye bajo licencia Free software y es sencillo de utilizar.

Desde el punto de vista de aprendizaje, lo ideal sería que el alumnado elaborase sus propios mapas conceptuales a partir de los apuntes que tienen a su disposición, pero esto requeriría un grado de conocimiento previo sobre la materia que no tienen. Parte del el equipo docente ya tiene cierta experiencia en la elaboración de mapas conceptuales y los ha incorporado a su docencia como material de apoyo complementario. Ahora pretendemos que los mapas conceptuales adquieran un papel principal en nuestra praxis docente y releguen a los apuntes a un papel secundario como material de apoyo para la creación de los mapas, desplazando en gran medida el aprendizaje memorístico por el significativo.

(cc) EY-NC-ND 2018, Universitat Politécnica de Valencia

Congreso IN-RED (2018) 


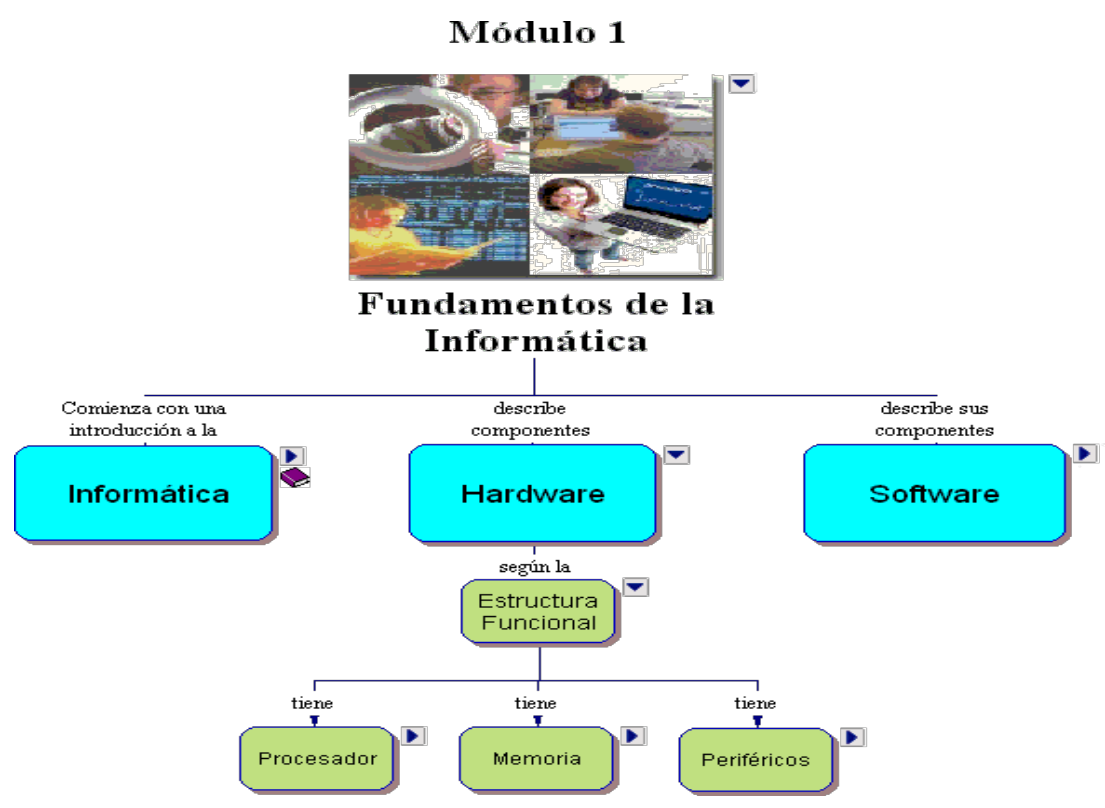

Fig. 2 Ejemplo de mapa conceptual

Para acometer esta tarea, el equipo docente está diseñando mapas conceptuales básicos, tipo esqueleto, con los principales conceptos y relaciones que deben contener en función del tema tratado (ver Figura 2). Estos mapas esqueleto deben servir de guía y ayuda a los estudiantes para que los completen y extiendan sirviéndose de los apuntes de la asignatura y de la utilidad de búsqueda de información disponible en la herramienta CmapTools. El trabajo de alumno se hará de forma individualizada. Las clases magistrales se usarán para explicar los mapas esqueleto y orientar a los alumnos en la elaboración mapas. Respecto a la evaluación, se tomará nota de la calidad de los mapas y del trabajo realizado en clase y se sumará a la nota obtenida en el examen final de la teoría.

\subsection{Aprendizaje cooperativo}

Respecto a la implementación del aprendizaje cooperativo, este va a ser puesto en marcha en la componente práctica de la asignatura. Como hemos indicado anteriormente, la mitad de los créditos de esta asignatura son créditos prácticos que se evalúan a partir de medir destreza en el uso de un equipo informático y serie de una serie aplicaciones informáticas, principalmente de ofimática. Estas destrezas se adquieren y entrenan en sesiones prácticas que se realizan en aulas de informática. Dependiendo de diversos factores (conocimientos previos, uso diario de la aplicaciones) nos encontramos con alumnos que suelen seguir las clases prácticas sin mayor problema y otros que les supone un verdadero hándicap. Este segundo grupo suele abandonar y/o suspender el examen práctico que se realiza a final del cuatrimestre.

A partir de las experiencias disponibles en la literatura, hemos diseñado un modelo de aprendizaje en el que los alumnos trabajan en pequeños grupos (3 o 4 estudiantes) con el objetivo común de mejorar las calificaciones individuales en el examen práctico. Los estudiantes se sientan juntos en el aula de informática y se apoyan los unos en los otros para realizar correctamente cada una de las prácticas. Consideraremos que el objetivo se ha 
alcanzado si todos los miembros del grupo alcanzan en la evaluación de las competencias prácticas una nota igual o superior a 6.5 puntos. Para dinamizar y tirar del grupo, se ha definido la figura de un líder que se encarga de emprender las iniciativas que considere oportunas para alcanzar el objetivo previsto. Si el objetivo se consigue, el líder será recompensado con un aumento de su calificación final en la asignatura. Si no es así, se le calificará con la nota obtenida de forma individual. La figura del líder va en la línea de cubrir la competencia de "liderazgo" recogida en el listado de competencias generales de la memoria del grado de la titulación y potenciar la capacidad de alumno para asumir responsabilidades y tomar decisiones.

La combinación de ambas herramientas ha de traducirse en una mejora de los resultados académicos así como de la puntuación obtenida en las encuestas de satisfacción del alumnado que anualmente se pasan sobre esta asignatura. Para la comparación usaremos los resultados disponibles de años anteriores.

\section{Resultados}

Como indicamos en la introducción, el proyecto de innovación docente ha empezado a ponerse en práctica este curso académico 2017-18. En lo que se refiere a los mapas conceptuales, actualmente estamos desarrollando los mapas esqueleto de cada uno de los temas, teniendo previsto entregárselos a los alumnos el siguiente curso 2018-19. Por lo tanto carecemos de datos para poder medir el impacto de los resultados de esta iniciativa.

Por el contrario, si que estamos muy satisfechos con las sensaciones y resultados de la puesta en marcha del aprendizaje cooperativo en las sesiones prácticas en aulas con ordenadores. Todos los años detectamos sombre un $15 \%$ de alumnos que abandonaban la asistencia a prácticas motivadas principalmente porque no son capaces de seguir el ritmo de las clases, bien porque se han incorporado tarde, bien por sus propias limitaciones en el trabajo con ordenadores. Sin embargo, este año no hemos detectado ningún abandono de la asignatura por estos motivos. De hecho hemos comprobado como alumnos, que de haberse mantenido el sistema anterior casi con total seguridad hubiesen abandonado la asignatura, este año no lo han hecho. Además, el clima de trabajo en la sesiones prácticas han cambiado sensiblemente a mejor. Los alumnos se muestran más activos y los que asumieron el papel de líderes han ejercido como tales. Se ha preocupado de que los miembros de sus equipos más rezagados aprendiesen a realizar las prácticas correctamente, juntándose en algunas ocasiones fuera del horario lectivo para realizar los guiones de prácticas. Como cabría esperar con estos cambios, los resultados académicos en la prueba práctica de la convocatoria de febrero han mejorado respecto a años anteriores (ver Figura 3). Así, podemos ver como han descendido el número de suspensos y no presentados y ha aumentado porcentualmente el número de sobresalientes y matrículas de honor.

(cc) EY-NC-ND 2018, Universitat Politécnica de Valencia

Congreso IN-RED (2018) 


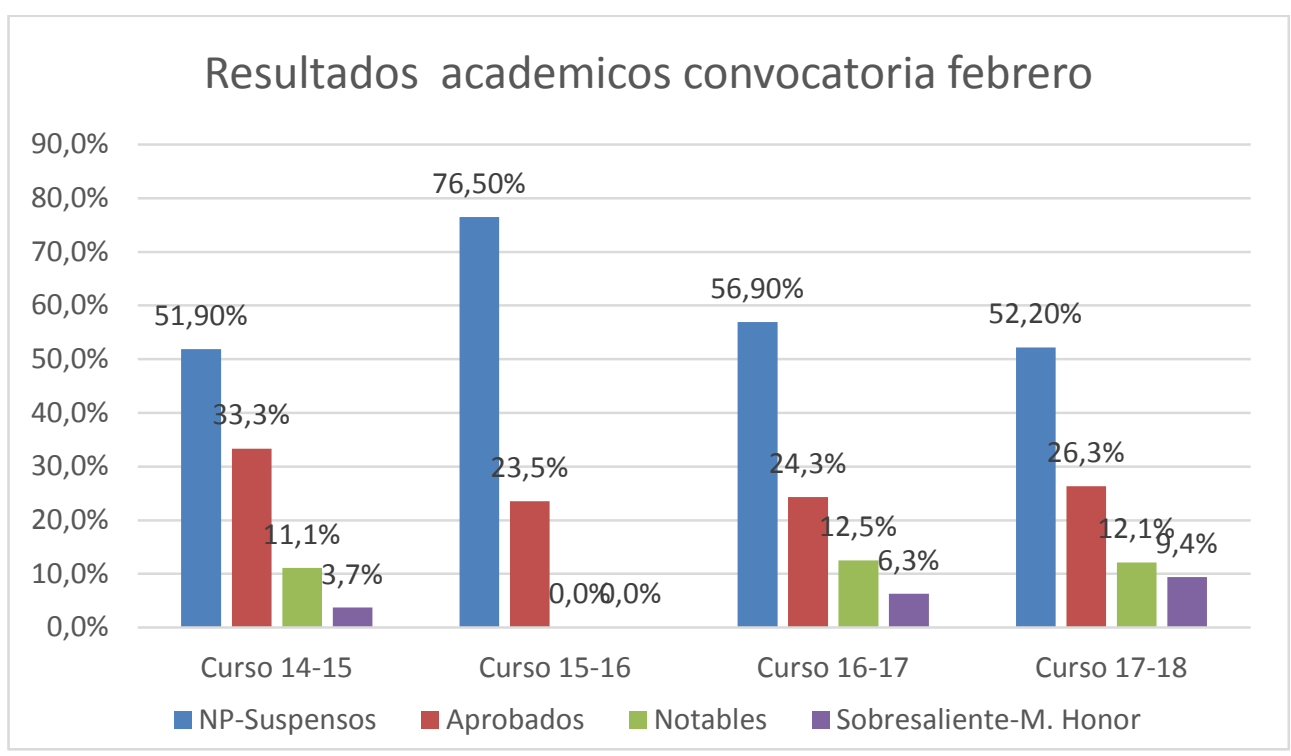

Fig. 3 Resultados académicos convocatoria de febrero

\section{Conclusiones}

En este trabajo hemos presentado las dos iniciativas de innovación docente que actualmente estamos desarrollado para mejorar las competencias y resultados académicos de una de las dos asignaturas de informática que se imparten en el grado en Gestión y Administración Pública. Esta asignatura viene obteniendo resultados insatisfactorios para el equipo docente que la imparte debido a dos motivos principales, i) la incorporación tardía al curso de una parte importante de estudiantes y ii) la dificultad en sí de los contenidos y la falta de destrezas en el manejo aplicaciones informáticas.

Para intentar paliar estos problemas, hemos propuesto incorporar como innovación docente el uso de mapas conceptuales para abordar los contenidos teóricos y el aprendizaje cooperativo para resolver el problema de la mejora de las destrezas prácticas. Vistas las peculiaridades de ambas metodologías, el equipo docente considera que su combinación puede ser una buena solución para intentar resolver los problemas detectados en esta asignatura y alcanzar los objetivos propuestos, además de cubrir varias de las competencias que suelen venir recogidas en las memorias de grado en cuanto a fomentar el trabajo en equipo, responsabilidad y liderazgo.

Desde el punto de vista de la transversalidad, entendemos que esta experiencia puede ser aplicada y/o extendida a otras asignaturas que se imparte en titulaciones con un perfil similar al grado en Gestión y Administración Pública (Derecho, Trabajo Social, Relaciones Laborales, ...), o en asignaturas con un contenido teórico complejo o en las que los resultados académicos de la parte práctica no sean los esperados y/o deseados.

\section{Agradecimientos.}

Este trabajo se enmarca dentro del Proyecto de Innovación Docente UJA PI2D-2017, correspondiente a la convocatoria de proyectos de innovación docente recogidos en el Plan 
Herramientas para la mejora de la práctica docente de la Informática. Experiencia piloto en el Grado en Gestión y Administración Pública

de Innovación e Incentivación de la Buenas Prácticas Docentes de la Universidad de Jaén 2016-2019.

\section{Referencias}

AUSUBEL, D. P. (1963). The psychology of meaningful verbal learning. New York: Grune and Stratton.

AUSUBEL, D. P. (1968). Educational psychology: A cognitive view. New York: Holt, Rinehart and Winston.

CAÑAS, A. J. et al. (2004). CmapTools: A knowledge modeling and sharing environment. Pamplona, Universidad Pública de Navarra, pp. 125-133.

GARCIA, R., TRAVER, J. y CANDELA, I. (2001). Aprendizaje cooperativo. Fundamentos, características y técnicas. Madrid: CCS.

I2D-UJA, (2016). "Plan de Innovación e Incentivación de las buenas prácticas docentes en la Universidad de Jaén (Plan I2D-UJA 2016)". [Online] Available at: https://www10.ujaen.es/conocenos/organos-gobierno/vicestudios/planinnovacion

JOHNSON, D. y JOHNSON, R. (1991). Learning Together and Alone. Cooperative, Competitive and Individualistic learning. 3rd ed. Needham Heights, Allyn and Bacon.

JOHNSON, D., JOHNSON, R. y HOLUBEC, E. (1999). El aprendizaje cooperativo en el aula. Buenos Aires: Paidós.

Novak, J. D. (1990). "Concept maps and vee diagrams: Two metacognitive tools for science and mathematics education". Instructional Science, Issue 19, pp. 29-52.

NOVAK, J. D. y MUSONDA, D., 1991. "A twelve-year longitudinal study of science concept learning". American Educational Research Journal, 28(1), pp. 117-153.

O'DONNELl, A., DANSEREAU, D. \& HALL, R. H. (2002). "Knowledge maps as scaffolds for cognitive processing". Educational Psychology Review, Issue 14, pp. 71-86.

PIAGET, J. (1973). La representación del Mundo en el Niño.. Madrid: Morata.

PRIETO, L. (2007). El aprendizaje cooperativo. Madrid: PPC.

Servicio de Innovación Educativa, 2008. Aprendizaje Cooperativo, s.l.: Universidad Politécnica de Madrid.

TAMAYO, M. F. A. (2006). "El mapa conceptual como una herramienta para aprender y enseñar". Plasticidad y Restauración Neurológica, 5(1), pp. 62-72.

WALES, J., (2018). "Apreder cómo aprendee es más importante que nunca". El pais. [Online]

Available at: http://aprendemosjuntos.elpais.com/especial/el-aprendizaje-informal-jimmywales/

(cc) EY-NC-ND 2018, Universitat Politécnica de Valencia

Congreso IN-RED (2018) 